\title{
Private Krankenversicherung
}

\section{So können Ärzte sparen}

— Wenn Ärzten die Beiträge für ihre private Krankenversicherung zu teuer werden, können sie beim gleichen Anbieter in einen günstigeren Tarif mit gleichem Leistungsumfang wechseln. Das ist meist sinnvoller als die Wahl eines neuen Anbieters, da die angesparten Alterungsrückstellungen nicht verloren gehen. Häufig sehen die Versicherer den Tarifwechsel im eigenen Haus aber nicht gern. Viele versuchen daher, Interessenten Steine in den Weg zu legen.

Timo Voss, vom Bund der Versicherten (BdV), empfiehlt Kunden, beim Versicherer schriftlich unter Verweis auf das Tarifwechselrecht Umstellungsangebote anzufordern. „Die Versicherten sollten den Brief per Einschreiben schicken und dem Versicherer eine Frist von 14 Tagen setzen." Reagiert der Versicherer auch auf Nachfragen nicht, sollte sich der Kunde direkt an den Vorstand wenden. Beim Prüfen der Angebote sollte der Versicherte auf mehrere Dinge achten, sagt Voss. Eine deut- liche Erhöhung des Selbstbehaltes sei nur sinnvoll, wenn es keine andere Alternative bei der Gesellschaft gebe. Kunden sollten geschlossene Tarife meiden, also Angebote, die Neukunden nicht mehr offenstehen. „In solchen Tarifen werden die Prämien in absehbarer Zeit steigen". Am besten informieren die Wechselwilligen die Versicherer von Anfang an darüber, dass sie keinen höheren Selbstbehalt und keinen geschlossenen Tarif wollen. „Fordern Sie aktuelle und verkaufsoffene Tarife vom Versicherer", rät der BdV. Er bietet Mitgliedern neben der Beratung auch aktive Unterstützung beim Tarifwechsel an. Dieser ExtraService kostet bis zu 400 Euro plus Steuern. Einige kommerzielle Anbieter wie Widge.de und beitragsoptimierung24.de haben sich auf das Geschäftsfeld Tarifwechsel spezialisiert. Entscheidet sich der Versicherte für einen neuen Tarif, erhalten sie einen Teil der erzielten Ersparnis: Bei Widge.de und dem Portal $1 \mathrm{~A}$ ist es zum Beispiel das Achtfache des eingespar- tes Monatsbeitrags. Für Harald Leissl, Vorstand der Delegare AG Ulm, die mit der Marke beitragsoptimierung24.de arbeitet, steht außer Frage, dass Kunden beim Tarifwechsel Unterstützung brauchen. Mehr als die Hälfte der Delegare-Kunden hätten bereits einen erfolglosen "Selbstversuch" hinter sich. Manche Versicherer würden wechselwillige Kunden gezielt in Tarife lenken, bei denen die Beitragsentwicklung in absehbarer Zeit problematisch werden könnte, so Leissl. Die Beratung der Versicherten in diesem schwierigen Feld sei aufwendig und rechtfertige das Honorar, betont er. Die Jahresersparnis der Kunden und damit die Vergütung des Unternehmens liege im Schnitt bei 2.500 Euro im Jahr.

Verbraucherschützer sehen die Aktivitäten der Wechsel-Spezialisten kritisch. Sie fürchten, dass die Unternehmen Kunden in Tarife lotsen, die zwar eine hohe Ersparnis bieten, aber keinen guten Versicherungsschutz.

Ilse Schlingesiepen 